\title{
An Inexact Update Method with Double Parameters for Nonnegative Matrix Factorization
}

\author{
Xiangli Li, ${ }^{1,2,3,4}$ Wen Zhang, ${ }^{1}$ Xiaoliang Dong, ${ }^{5}$ and Juanjuan Shi ${ }^{1}$ \\ ${ }^{1}$ School of Mathematics and Computing Science, Guilin University of Electronic Technology, Guilin 541004, China \\ ${ }^{2}$ Guangxi Key Laboratory of Automatic Detecting Technology and Instruments, Guilin University of Electronic Technology, \\ Guilin, Guangxi 541004, China \\ ${ }^{3}$ Guangxi Colleges and Universities Key Laboratory of Data Analysis and Computation, Guilin University of Electronic Technology, \\ Guilin, Guangxi 541004, China \\ ${ }^{4}$ Guangxi Key Laboratory of Cryptography and Information Security, Guilin University of Electronic Technology, \\ Guilin, Guangxi 541004, China \\ ${ }^{5}$ School of Mathematics and Information, Beifang University of Nationalities, Yinchuan 710021, China
}

Correspondence should be addressed to Xiangli Li; lixiangli@guet.edu.cn

Received 14 July 2016; Revised 15 October 2016; Accepted 16 October 2016

Academic Editor: Elisa Francomano

Copyright (C) 2016 Xiangli Li et al. This is an open access article distributed under the Creative Commons Attribution License, which permits unrestricted use, distribution, and reproduction in any medium, provided the original work is properly cited.

Nonnegative matrix factorization (NMF) has been used as a powerful date representation tool in real world, because the nonnegativity of matrices is usually required. In recent years, many new methods are available to solve NMF in addition to multiplicative update algorithm, such as gradient descent algorithms, the active set method, and alternating nonnegative least squares (ANLS). In this paper, we propose an inexact update method, with two parameters, which can ensure that the objective function is always descent before the optimal solution is found. Experiment results show that the proposed method is effective.

\section{Introduction}

Nonnegative matrix factorization (NMF) [1] is not only a well-known matrix decomposition approach but also an utility and efficient feature extraction technique. NMF was first put forward by Lee and Seung. Recently, NMF has been successfully applied in many fields, including face verification [2], text mining [3], gene expression data [4], blind source separation [5], and signal processing [6].

A nonnegative matrix $A \in R_{+}^{m \times n}$ is decomposed into two low-rank nonnegative matrices $W \in R_{+}^{m \times r}$ and $H \in R_{+}^{r \times n}$ such that $A$ is approximately equal to $W H$, denoted by

$$
A \approx W \times H,
$$

where $r \in N_{+}$and $r \ll m n /(m+n)$. $W$ and $H$ mean different things to different applications; for example, in blind source separation (BSS), $W$ and $H$ are called mixing matrix and source signal matrix, respectively.

In order to decrease the approximation error between $A$ and $W H$, the Euclidean distance-based model is employed in this paper. Namely, NMF can be expressed as the following optimization form:

$$
\begin{array}{ll}
\min _{W, H} & F(W, H)=\frac{1}{2}\|A-W H\|_{F}^{2}, \\
\text { s.t. } & W \geq 0, \\
& H \geq 0,
\end{array}
$$

where $\|\cdot\|_{F}$ is the Frobenius norm and $W \geq 0(H \geq 0)$ means that all elements of the matrix $W(H)$ are nonnegative. Clearly, it is difficult to find a global optimal solution $(W, H)$ because the objective function $F(W, H)$ is nonconvex. Therefore, the remaining issue is how to solve the nonconvex problem.

In 2001, Lee and Seung tried to find a local optimal solution instead of a global one and proposed multiplicative update algorithm [7]. Multiplicative update algorithm is widely used as an efficient computational method for NMF. The update rules for (2) are given as follows: 


$$
\begin{gathered}
H_{i j}^{k+1}=H_{i j}^{k} \frac{\left(\left(W^{k}\right)^{T} V\right)_{i j}}{\left(\left(W^{k}\right)^{T} W^{k} H^{k}\right)_{i j}}, \\
W_{i j}^{k+1}=W_{i j}^{k} \frac{V\left(\left(H^{k+1}\right)^{T}\right)_{i j}}{\left(W^{k} H^{k+1}\left(H^{k+1}\right)^{T}\right)_{i j}},
\end{gathered}
$$

where $k$ represents the iteration count. Later, many new methods were available to solve NMF in addition to multiplicative update algorithm, such as gradient descent algorithms $[8,9]$, the active set method [10], and alternating nonnegative least squares (ANLS) [11-13].

For the sake of solving the minimum problem (2), Hien et al. [14] have proposed a novel algorithm, in which the update rule contains only a parameter and the parameter selection is considered exact in some cases. Compared with multiplicative update algorithm, the novel algorithm [14] has fast convergence speed and small decomposition error.

Some inexact technique is widely applied to large scale optimization problems. Based on this idea, we employ an inexact parameter instead of the exact parameter [14]. In the meantime, we add another parameter to accelerate the speed decrease of objective function. Hence, we present an inexact update algorithm with two parameters. The proposed method also updates the elements of matrices $W$ and $H$ one by one. Under some assumptions, the proposed method ensures that the objective function is always descent before the optimal solution is found.

Similar to multiplicative update algorithm, the proposed method has many advantages, including its efficient storage, simple calculation, and good results. In multiplicative update algorithm, the descent property is established by means of an auxiliary function. However, the proposed method is descent by the local monotonicity of a quadratic function. And it has a faster convergent speed. Besides, the main idea of ANLS is that two optimization problems can be solved alternately. By contrast, the proposed method is easier to implement.

The remainder of this paper is organized as follows: we present the procedure to update an element of the matrices $W$ and $H$ in Section 2. In Section 3, we give an inexact update method with double parameters for NMF and establish the convergent properties. In Section 4, experimental results demonstrate the validity of the method. Finally, we conclude this paper.

\section{Algorithm for Updating an Element of Matrix}

In this section, we will discuss the procedure to update an element of the matrices $W$ and $H$. In [14], $W_{i j}$ is adjusted by adding a parameter $\alpha$ :

$$
\widetilde{W}_{i j}=W_{i j}+\alpha
$$

where

$$
\begin{aligned}
& \alpha= \begin{cases}0, & \text { if } q=0, \\
\max \left(-\frac{q}{p},-W_{i j}\right), & \text { if } q>0, \\
-\frac{q}{p}, & \text { if } q<0,\end{cases} \\
& p=\sum_{b=1}^{m} H_{j b}^{2}, \\
& q=\sum_{b=1}^{m}(W H-V)_{i b} \times H_{j b} .
\end{aligned}
$$

Motivated by the above work, we give the following adjustment to $W_{i j}$ by two parameters $\alpha$ and $\beta$ :

$$
\widetilde{W}_{i j}=\beta W_{i j}+\alpha,
$$

in which $\beta$ can be viewed as a constant as well as a function. We define the parameter $\alpha$ by a certain value $\beta$, where $0<$ $\beta \leq 1$. Similar to [14], we have that if $a \neq i$,

$$
(\widetilde{W} H)_{a b}=(W H)_{a b}
$$

otherwise,

$$
\begin{aligned}
(\widetilde{W} H)_{i b} & =\sum_{p=1}^{r} \widetilde{W}_{i p} H_{p b}=\sum_{p \neq j} W_{i p} H_{p b}+\widetilde{W}_{i j} H_{j b} \\
& =\sum_{p \neq j} W_{i p} H_{p b}+\beta W_{i j} H_{j b}+\alpha H_{j b} \\
& =(W H)_{i b}+(\beta-1) W_{i j} H_{j b}+\alpha H_{j b} .
\end{aligned}
$$

Next, we deduce

$$
\begin{aligned}
f(\widetilde{W}, H)= & \frac{1}{2} \sum_{a} \sum_{b}\left[(\widetilde{W} H)_{a b}-V_{a b}\right]^{2} \\
= & \frac{1}{2} \sum_{a \neq i} \sum_{b}\left[(W H)_{a b}-V_{a b}\right]^{2} \\
& +\frac{1}{2} \sum_{b}\left[(\widetilde{W} H)_{i b}-V_{i b}\right]^{2} \\
= & f(W, H)+g(\alpha),
\end{aligned}
$$

where

$$
\begin{aligned}
g(\alpha) & =\frac{1}{2}\left[\alpha+(\beta-1) W_{i j}\right]^{2} p+\left[\alpha+(\beta-1) W_{i j}\right] q, \\
p & =\sum_{b} H_{j b}^{2}, \\
q & =\sum_{b}(W H-V)_{i b} \times H_{j b} .
\end{aligned}
$$

In order to ensure that the function $f(\widetilde{W}, H)$ possesses the descent property, we should define $\alpha$ so that $g(\alpha)$ is 
nonpositive in $\widetilde{W}_{i j}=\beta W_{i j}+\alpha \geq 0$. For a given value $\beta, \alpha$ is expressed as follows:

$$
\alpha= \begin{cases}(1-\beta) W_{i j}, & \text { if } q=0, \\ \max \left\{(1-\beta) W_{i j}-\gamma,-\beta W_{i j}\right\}, & \text { if } q>0, \\ (1-\beta) W_{i j}-\gamma, & \text { if } q<0 .\end{cases}
$$

Since $g(\alpha)$ is a quadratic function, $\gamma \in(0,-2 q / p)$ or $\gamma \in$ $(-2 q / p, 0)$. In other words, the $\gamma$ value can be "inexact." If $\gamma=-q / p$, specially, $g(\alpha)$ reaches the minimum value. It is obvious that the decline of $f(\widetilde{W}, H)$ is the steepest; at the moment, $\alpha$ is believed to be "exact."

Lemma 1. If $\widetilde{W}_{i j}$ does not satisfy the KKT conditions, then $f(\widetilde{W}, H)<f(W, H)$; otherwise, $\widetilde{W}=W$; this implies $f(\widetilde{W}$, $H)=f(W, H)$.

Proof. Clearly, $W_{i j} \geq 0,\left((W H-V) H^{T}\right)_{i j} \geq 0$, and $W_{i j} \times$ $\left(\left((W H-V) H^{T}\right)\right)_{i j}=0$ are the KKT conditions of (2). By the definition of $q$, we have

$$
W_{i j} \times q=0, \quad W_{i j} \geq 0, q \geq 0 .
$$

If $\widetilde{W}_{i j}$ does not satisfy (12), we can obtain $g(\alpha)<0$ in the condition of $q<0$ or $q>0$ and $W_{i j}>0$. Therefore, $f(\widetilde{W}$, $H)<f(W, H)$.

Conversely, if $q=0$ and $\alpha=(1-\beta) W_{i j}$, we have $\widetilde{W}=W$; if $q>0$ and $W_{i j}=0$, it is easy to see that $\alpha=0$ and $\widetilde{W}=W=\mathbf{0}$. In either case, $f(\widetilde{W}, H)=f(W, H)$.

Similarly, we can deduce the following update rule of $H$ and Lemma 2:

$$
\widetilde{H}_{i j}=\beta^{\prime} H_{i j}+\alpha^{\prime}
$$

Let

$$
\begin{aligned}
& u=\sum_{a} W_{a i}^{2}, \\
& v=\sum_{a} W_{a i} \times(W H-V)_{a j} .
\end{aligned}
$$

For a given value $\beta^{\prime}, \alpha^{\prime}$ is expressed as follows:

$$
\alpha^{\prime}= \begin{cases}\left(1-\beta^{\prime}\right) H_{i j}, & \text { if } q=0, \\ \max \left\{\left(1-\beta^{\prime}\right) H_{i j}-\gamma^{\prime},-\beta^{\prime} H_{i j}\right\}, & \text { if } q>0, \\ \left(1-\beta^{\prime}\right) H_{i j}-\gamma^{\prime}, & \text { if } q<0,\end{cases}
$$

where $\gamma^{\prime} \in(0,-2 v / u)$ or $\gamma^{\prime} \in(-2 v / u, 0)$.

Lemma 2. If $\widetilde{H}_{i j}$ does not satisfy the KKT conditions, then $f(W, \widetilde{H})<f(W, H)$; otherwise, $\widetilde{H}=H$; this implies $f(W$, $\widetilde{H})=f(W, H)$.

\section{The Proposed Algorithm}

Based on the above analysis, in this section, we report our algorithm as follows.
Algorithm 3.

(s.1) Give the starting point $W^{1} \geq 0$ and $H^{1} \geq 0$; set $k=1$.

(s.2) Update $W^{k}$ by using the following formula:

$$
\begin{array}{r}
\text { For } j=1, \ldots, r \text { and } i=1, \ldots, n \\
\qquad \widetilde{W}_{i j}=\beta W_{i j}+\alpha .
\end{array}
$$

$\alpha$ is computed from (11).

(s.3) Update $H^{k}$ by using the following formula:

$$
\begin{array}{r}
\text { For } i=1, \ldots, r \text { and } i=1, \ldots, m \\
\qquad \widetilde{H}_{i j}=\beta^{\prime} H_{i j}+\alpha^{\prime} .
\end{array}
$$

$\alpha^{\prime}$ is computed from (15).

(s.4) Let $k=k+1$. Go to (s.2).

In order to ensure the monotonous decrease of $f(W, H)$, we give the next theorem, which follows directly from Lemmas 1 and 2 and Algorithm 3.

Theorem 4. Suppose that $\left(W^{k}, H^{k}\right)$ is generated by Algorithm 3; then $f\left(W^{k}, H^{k}\right)$ is monotonically decreasing; that is,

$$
f\left(W^{k+1}, H^{k+1}\right)<f\left(W^{k}, H^{k}\right) .
$$

Corollary 5. Sequence $f\left(W^{k}, H^{k}\right)$ is bounded; then $f\left(W^{k}\right.$, $\left.H^{k}\right)$ is a convergence sequence; namely, there exists a positive constant $C$ such that

$$
\lim _{k \rightarrow \infty} f\left(W^{k}, H^{k}\right)=C
$$

for all $k$ large enough.

It is clear to obtain the above corollary from theorem. Next, the another convergence property of Algorithm 3 is given.

Theorem 6. Suppose $(\bar{W}, \bar{H})$ is a limit point of $\left(W^{k}, H^{k}\right)$ and

$$
\begin{aligned}
& \sum_{b=1}^{m} \bar{H}_{j b}^{2}>0, \quad j=1, \ldots, r, \\
& \sum_{a=1}^{n} \bar{W}_{a i}^{2}>0, \quad i=1, \ldots, r .
\end{aligned}
$$

Then $(\bar{W}, \bar{H})$ is the stationary point of problem (2).

Since the above theorem corresponds to [14] (Theorem 3.2.2) and the proof is the same as that in [14] (Theorem 3.2.2), we will not prove it here.

\section{Numerical Experiments}

In this section, we give some numerical experiments of Algorithm 3 and compare its behavior with the method of [14] 
TABLE 1: $A=a b s(\operatorname{rand} n(m, r)) \times a b s(\operatorname{rand} n(r, n))$ and $\beta=\beta^{\prime}=1 / 2$.

\begin{tabular}{|c|c|c|c|c|c|}
\hline$(m, n, r)$ & & Iter & Fun & Time & $\Delta(W, H)$ \\
\hline \multirow{2}{*}{$(50,50,4)$} & Algorithm 3 & 2585 & $1.239 e-016$ & 6.989 & $2.548 e-009$ \\
\hline & NNMF & 5021 & $1.523 e-014$ & 13.104 & $2.556 e-009$ \\
\hline \multirow{2}{*}{$(100,50,5)$} & Algorithm 3 & 2448 & $1.808 e-017$ & 13.037 & $1.342 e-009$ \\
\hline & NNMF & 7074 & $5.450 e-015$ & 36.535 & $1.358 e-009$ \\
\hline \multirow{2}{*}{$(100,100,5)$} & Algorithm 3 & 6970 & $1.689 e-017$ & 47.876 & $1.011 e-009$ \\
\hline & NNMF & 7506 & $3.889 e-015$ & 59.702 & $1.010 e-009$ \\
\hline \multirow{2}{*}{$(200,200,4)$} & Algorithm 3 & 4451 & $8.486 e-018$ & 78.766 & $6.261 e-010$ \\
\hline & NNMF & 18033 & $2.282 e-015$ & 198.386 & $6.275 e-010$ \\
\hline \multirow{2}{*}{$(200,200,8)$} & Algorithm 3 & 18930 & $1.860 e-021$ & 385.494 & $3.149 e-010$ \\
\hline & NNMF & 19459 & $1.015 e-015$ & 391.797 & $3.148 e-010$ \\
\hline \multirow{2}{*}{$(200,300,6)$} & Algorithm 3 & 14356 & $1.150 e-017$ & 295.138 & $3.348 e-010$ \\
\hline & NNMF & 20542 & $1.335 e-015$ & 424.479 & $3.351 e-010$ \\
\hline \multirow{2}{*}{$(500,100,20)$} & Algorithm 3 & 14356 & $1.150 e-017$ & 295.138 & $3.348 e-010$ \\
\hline & NNMF & 20542 & $1.335 e-015$ & 424.479 & $3.351 e-010$ \\
\hline
\end{tabular}

(NNMF). All codes of the computer procedures are written in MATLAB and run in a MATLAB 7.10 and are carried out on a PC (CPU $2.13 \mathrm{GHz}, 2 \mathrm{G}$ memory) with Windows 7 operation system environment.

In experiments, we will compare the following statistics: the number of iterations (Iter), the CPU time in second (time), the speed of convergence to stationary point, and the minimum value of objective function value (fun). Same as [14], the speed of convergence is measured by using the following formula:

$$
\Delta(W, H)=\frac{\delta(W, H)}{\delta(W)+\delta(H)},
$$

where

$\Delta(W, H)$

$$
\begin{aligned}
= & \sum_{i=1}^{n} \sum_{a=1}^{r}\left|\min \left(W_{i a},\left((W H-V) H^{T}\right)_{i a}\right)\right| \\
& +\sum_{b=1}^{r} \sum_{j=1}^{m}\left|\min \left(H_{b j},\left(W^{T}(W H-V)\right)_{b j}\right)\right| .
\end{aligned}
$$

$\delta(W)$ is the number of elements of the set

$$
\begin{aligned}
& \left\{\left|\min \left(W_{i a},\left((W H-V) H^{T}\right)_{i a}\right)\right| \neq 0, i=1, \ldots, n, a\right. \\
& \quad=1, \ldots, r\}
\end{aligned}
$$

and $\delta(H)$ is the number of elements of the set

$$
\begin{aligned}
& \left\{\left|\min \left(H_{b j},\left(W^{T}(W H-V)\right)_{b j}\right)\right| \neq 0, j\right. \\
& \quad=1, \ldots, m, b=1, \ldots, r\} .
\end{aligned}
$$

$\Delta(W, H)$ is called a normalized KKT residual.
In order to avoid the initial point effect on the numerical result, in every experiment, we use 20 initial points, and every initial point $\left(W^{1}, H^{1}\right)$ is randomly generated. We list the average values of Iter, Fun, Time, and $\Delta(W, H)$, respectively.

In Table 1, the relevant parameters of Algorithm 3 are specified as follows:

$$
\begin{aligned}
& \beta=\beta^{\prime}=\frac{1}{2}, \\
& \gamma=-1.6 \frac{q}{p} \\
& \gamma^{\prime}=-1.6 \frac{v}{u} .
\end{aligned}
$$

From Table 1, we can clearly see the following two points: (1) Algorithm 3 requires fewer iterations and fewer function factorization errors and is less time consuming; (2) for the speed of convergence, Algorithm 3 is not less than NNMF.

In Table 2, the relevant parameters of Algorithm 3 are specified as follows:

$$
\begin{aligned}
& \beta=\beta^{\prime}=1, \\
& \gamma=-1.6 \frac{q}{p}, \\
& \gamma^{\prime}=-1.6 \frac{v}{u} .
\end{aligned}
$$

From Table 2, we find that Algorithm 3 is still better than NNMF for most of the test problems. Note that Algorithm 3 differs from the method of [14] only in the values of $\gamma$ and $\gamma^{\prime}$, in which one is "inexact" and another is "exact." Moreover, from Tables 1 and 2, the value of Fun in Table 1 is more efficient than Table 2 for Algorithm 3. 
TABLE 2: $A=a b s(\operatorname{rand} n(m, r)) \times a b s(\operatorname{rand} n(r, n))$ and $\beta=\beta^{\prime}=1$.

\begin{tabular}{lccccc}
\hline$(m, n, r)$ & & Iter & Fun & Time & $\Delta(W, H)$ \\
\hline$(50,50,4)$ & Algorithm 3 & 2382 & $2.429 e-015$ & 6.022 & $2.526 e-009$ \\
& NNMF & 4157 & $1.106 e-014$ & $2.546 e-009$ \\
$(100,50,5)$ & Algorithm 3 & 7556 & $4.610 e-015$ & 30.280 & $1.356 e-009$ \\
& NNMF & 14323 & $1.624 e-014$ & 54.663 & 95.036 \\
$(200,100,10)$ & Algorithm 3 & 4572 & $1.480 e-016$ & 141.275 & $3.355 e-009$ \\
& NNMF & 8171 & $4.707 e-015$ & 107.563 & $3.380 e-010$ \\
$(200,200,4)$ & Algorithm 3 & 9942 & $7.142 e-016$ & 162.257 & $6.277 e-010$ \\
& NNMF & 15854 & $2.482 e-015$ & 272.971 & $3.279 e-010$ \\
$(200,300,6)$ & Algorithm 3 & 22006 & $4.955 e-016$ & 453.043 & $3.350 e-010$ \\
& NNMF & 13113 & $1.396 e-015$ & & \\
\hline
\end{tabular}

TABLE 3: $A=a b s(\operatorname{rand} n(m, r)) \times a b s(\operatorname{rand} n(r, n)), \beta=e^{-k / m}$, and $\beta^{\prime}=e^{-k / n}$.

\begin{tabular}{|c|c|c|c|c|c|}
\hline$(m, n, r)$ & & Iter & Fun & Time & $\Delta(W, H)$ \\
\hline \multirow{2}{*}{$(50,50,4)$} & Algorithm 3 & 1635 & $1.438 e-015$ & 4.852 & $2.525 e-009$ \\
\hline & NNMF & 3896 & $1.318 e-014$ & 10.218 & $2.540 e-009$ \\
\hline \multirow{2}{*}{$(100,50,5)$} & Algorithm 3 & 2833 & $6.945 e-016$ & 13.588 & $1.350 e-009$ \\
\hline & NNMF & 6823 & $7.085 e-015$ & 31.309 & $1.354 e-009$ \\
\hline \multirow{2}{*}{$(100,100,5)$} & Algorithm 3 & 3835 & $6.004 e-016$ & 25.241 & $1.009 e-009$ \\
\hline & NNMF & 7168 & $3.711 e-015$ & 44.616 & $1.012 e-009$ \\
\hline \multirow{2}{*}{$(200,200,4)$} & Algorithm 3 & 10703 & $3.491 e-016$ & 104.272 & $6.272 e-010$ \\
\hline & NNMF & 16664 & $2.878 e-015$ & 179.542 & $6.274 e-010$ \\
\hline \multirow{2}{*}{$(200,200,8)$} & Algorithm 3 & 15445 & $1.220 e-016$ & 207.822 & $3.139 e-010$ \\
\hline & NNMF & 20571 & $1.358 e-015$ & 399.597 & $3.153 e-010$ \\
\hline \multirow{2}{*}{$(200,300,6)$} & Algorithm 3 & 10973 & $1.930 e-016$ & 200.539 & $3.348 e-010$ \\
\hline & NNMF & 21923 & $1.907 e-015$ & 440.734 & $3.351 e-010$ \\
\hline
\end{tabular}

In Table 3, the relevant parameters of Algorithm 3 are specified as follows:

$$
\begin{aligned}
& \beta=e^{-k / m}, \\
& \beta^{\prime}=e^{-k / n}, \\
& \gamma=-1.6 \frac{q}{p}, \\
& \gamma^{\prime}=-1.6 \frac{v}{u} .
\end{aligned}
$$

In Table 3, $\beta$ and $\beta^{\prime}$ are regarded as a function, respectively. It is obvious that average numbers of iterations and the minimum value of objective function value for Algorithm 3 are smaller than those for NNMF, CPU time of Algorithm 3 is less than that of NNMF, and the speed of convergence of Algorithm 3 and NNMF is very close.

In Figure 1, we compare the performance of the proposed method with NNMF, respectively. The convergence curves are given as follows.

From Figure 1, it is obvious that the speed of decrease of the objective function value is faster for Algorithm 3. And the numbers of iterations for Algorithm 3 are smaller than that for NNMF.

For all test problems, although the two methods have the same speed of convergence to stationary point, the proposed method requires fewer iterations and fewer function factorization errors and is less time consuming. Consequently, the above experiment results show that Algorithm 3 performs better than the method of [14] by choosing the parameters $\beta$ and $\gamma$ suitably.

\section{Conclusions}

In this paper, we propose an inexact update method, with two parameters, which can ensure that the objective function is always descent before the optimal solution is found. Experiment results show that the proposed method performs better than the method of [14].

\section{Competing Interests}

The authors declare that they have no competing interests.

\section{Acknowledgments}

This project was supported by the National Natural Science Foundation of China (Grants nos. 61362021, 11361018, and 11601012), Guangxi Natural Science Foundation (nos. 2014GXNSFAA118003, 2014GXNSFFA118001), the Scientific Research Foundation of the Higher Education Institutions 

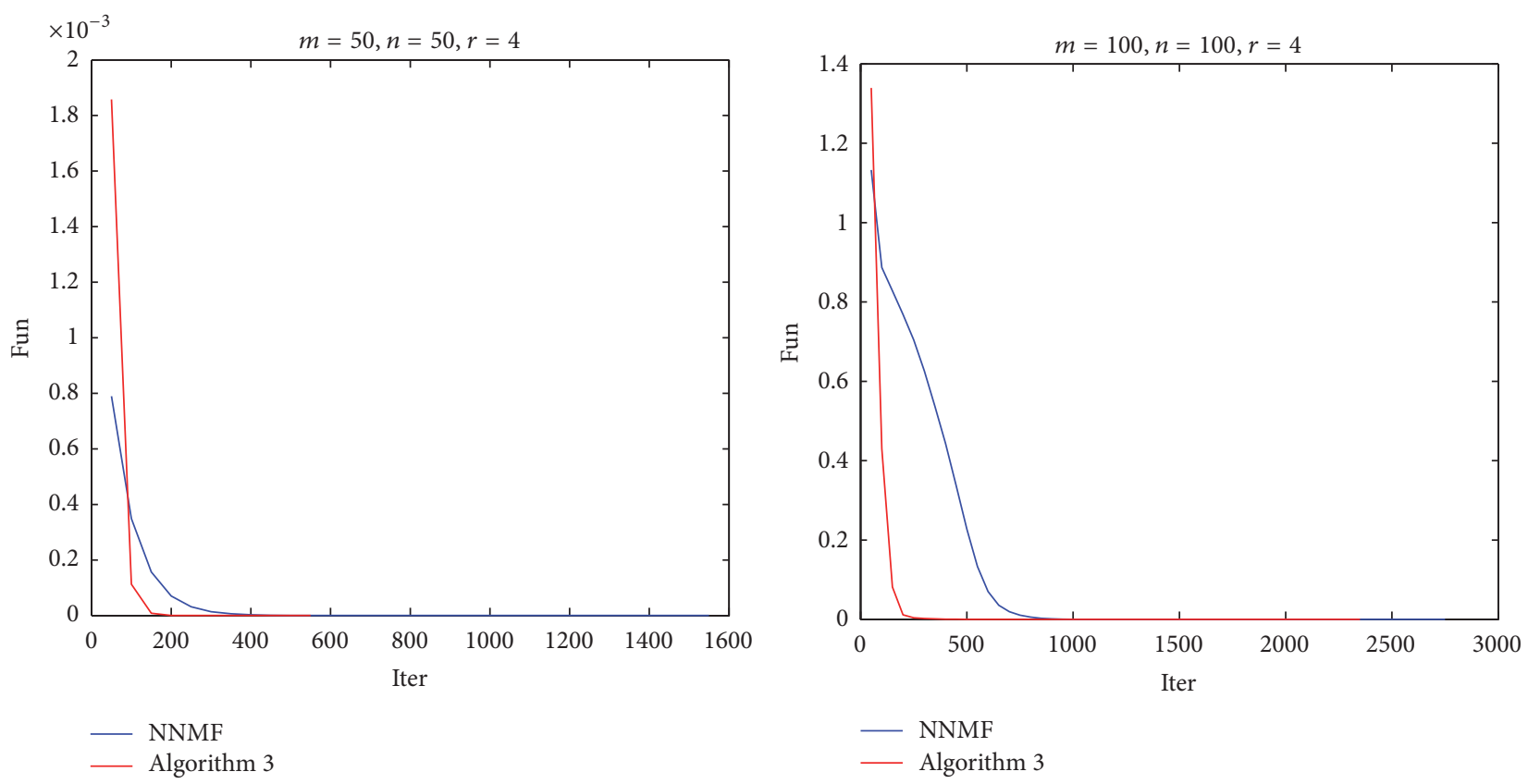

FIGURE 1: Performance profiles.

of Guangxi (Grant no. ZD2014050), Scientific Research Program Funded by Shaanxi Provincial Education Department (no. 16JK1435), and Guangxi Key Laboratory of Automatic Detecting Technology and Instruments (no. YQ16112).

\section{References}

[1] D. D. Lee and H. S. Seung, "Learning the parts of objects by non-negative matrix factorization," Nature, vol. 401, no. 6755, pp. 788-791, 1999.

[2] S. Zafeiriou, A. Tefas, I. Buciu, and I. Pitas, "Exploiting discriminant information in nonnegative matrix factorization with application to frontal face verification," IEEE Transactions on Neural Networks, vol. 17, no. 3, pp. 683-695, 2006.

[3] V. P. Pauca, F. Shahnaz, M. W. Berry, and R. J. Plemmons, "Text mining using non-negative matrix factorizations," in Proceedings of the 4th SIAM International Conference on Data Mining, pp. 452-456, 2004.

[4] P. M. Kim and B. Tidor, "Subsystem identification through dimensionality reduction of large-scale gene expression data," Genome Research, vol. 13, no. 7, pp. 1706-1718, 2003.

[5] A. Cichocki, R. Zdunek, and S.-I. Amari, "New algorithms for non-negative matrix factorization in applications to blind source separation," in Proceedings of the IEEE International Conference on Acoustics, Speech and Signal Processing (ICASSP '06), pp. V621-V624, May 2006.

[6] B. Gao, W. L. Woo, and S. S. Dlay, "Variational regularized 2-D nonnegative matrix factorization," IEEE Transactions on Neural Networks and Learning Systems, vol. 23, no. 5, pp. 703-716, 2012.

[7] D. D. Lee and H. S. Seung, "Algorithms for non-negative matrix factorization," in Proceedings of the 14th Annual Neural Information Processing Systems Conference (NIPS '00), vol. 13, pp. 556-562, Denver, Colo, USA, December 2000.

[8] N. Guan, D. Tao, Z. Luo, and B. Yuan, "Manifold regularized discriminative nonnegative matrix factorization with fast gradient descent," IEEE Transactions on Image Processing, vol. 20, no. 7, pp. 2030-2048, 2011.

[9] Z. Dai, X. Chen, and F. Wen, “A modified Perry's conjugate gradient method-based derivative-free method for solving largescale nonlinear monotone equations," Applied Mathematics and Computation, vol. 270, pp. 378-386, 2015.

[10] H. Kim and H. Park, "Nonnegative matrix factorization based on alternating nonnegativity constrained least squares and active set method," SIAM Journal on Matrix Analysis and Applications, vol. 30, no. 2, pp. 713-730, 2008.

[11] P. Paatero and U. Tapper, "Positive matrix factorization: a nonnegative factor model with optimal utilization of error estimates of data values," Environmetrics, vol. 5, no. 2, pp. 111-126, 1994.

[12] Z. Dai, D. Li, and F. Wen, "Worse-case conditional value-at-risk for asymmetrically distributed asset scenarios returns," Journal of Computational Analysis and Applications, vol. 20, no. 2, pp. 237-251, 2016.

[13] F. Wen, Z. He, Z. Dai, and X. Yang, "Characteristics of investors' risk preference for stock markets," Economic Computation and Economic Cybernetics Studies and Research, vol. 48, no. 3, pp. 235-254, 2015.

[14] T. D. Hien, D. Van Tuan, P. V. At, and L. H. Son, "Novel algorithm for non-negative matrix factorization," New Mathematics and Natural Computation, vol. 11, no. 2, pp. 121-133, 2015. 


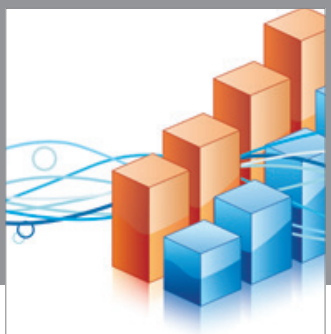

Advances in

Operations Research

vatem alat4

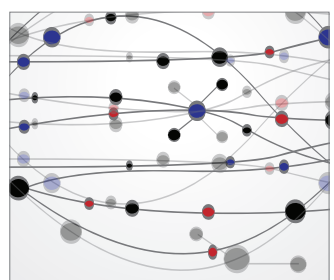

\section{The Scientific} World Journal
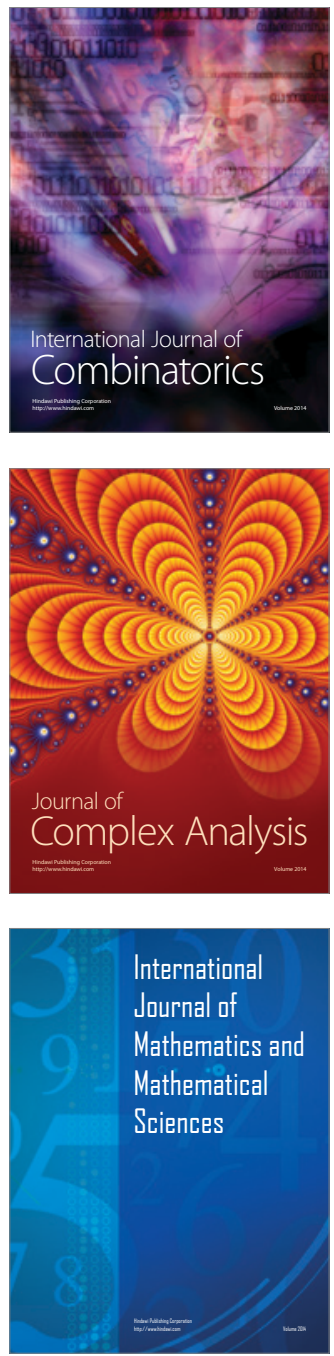
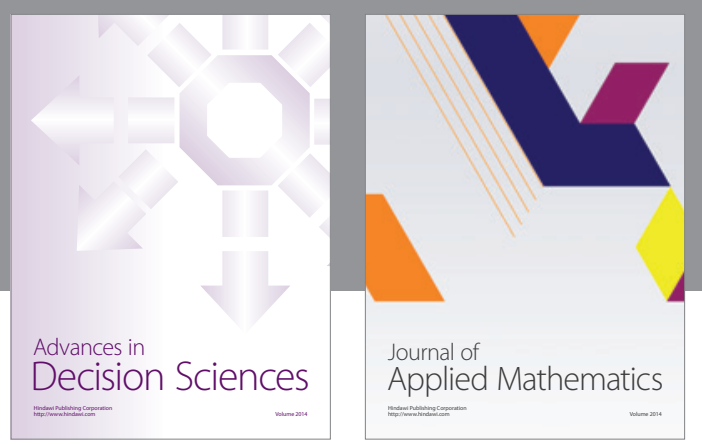

Algebra

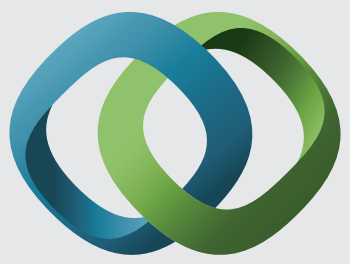

\section{Hindawi}

Submit your manuscripts at

http://www.hindawi.com
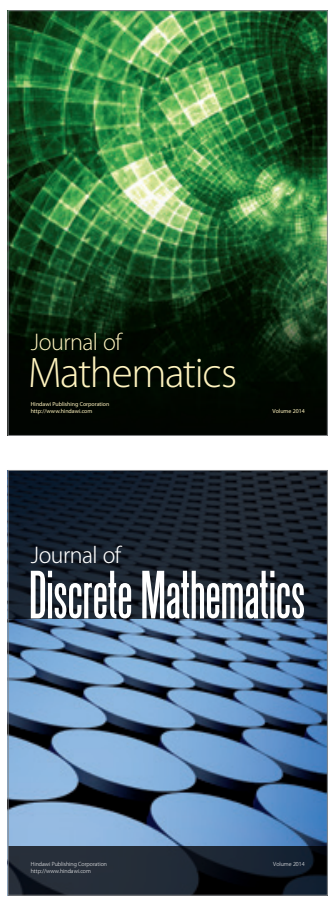

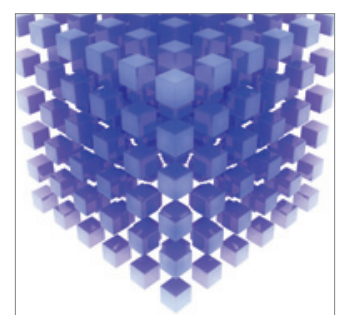

Mathematical Problems in Engineering
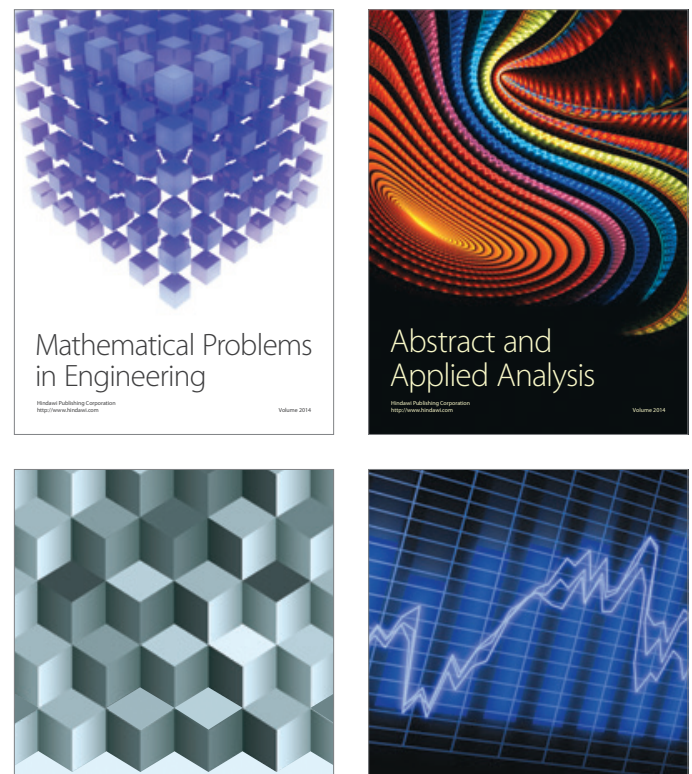

Journal of

Function Spaces

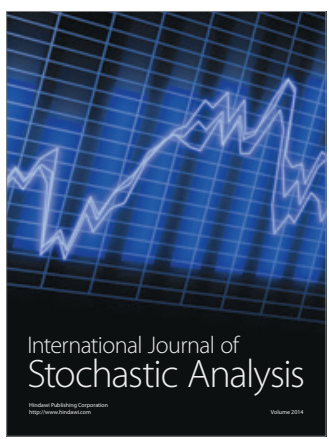

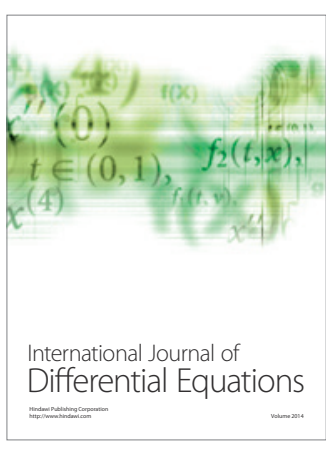
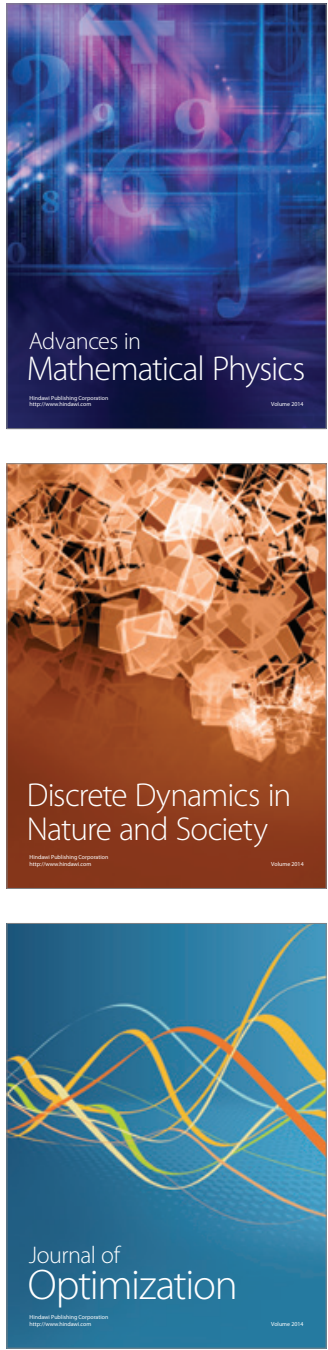